\title{
Performance of Mobility Models with different Routing Protocols by using Simulation Tools for WSN: A Review
}

\author{
Megha Jain ${ }^{1}$, V. K. Patle ${ }^{2}$, Sanjay Kumar ${ }^{3}$ \\ School of Studies in Computer Science \& I.T, Pt. Ravi Shankar Shukla University, Raipur, Chhattisgarh, India ${ }^{1,2,3}$
}

\begin{abstract}
Research on the mobility models for wireless sensor networks has been an interesting area of networking. As a result, researchers over the past few years have measured the performance of mobility models by applying protocols through various simulation tools for wireless sensor networks. Wireless sensor network is a group of sensing devices which are geographically distributed that monitor the condition around it such as physical or environmental which communicates wirelessly to cooperatively pass data to the main location. The goal of the proposed work is to provide researchers with clear idea about the available mobility models for wireless sensor network performance which can be investigated on various available simulation tools with several metrics of sensor networks. Hence, we attempt to provide an overview of the current research status for wireless sensor network mobility models.
\end{abstract}

Keywords: Mobility Model, WSN, Performance Metrics, Simulation Tools, Routing Protocols.

\section{INTRODUCTION}

Mobility models call movement of users, change in their location, velocity and acceleration over time. Such models are simulated with the investigated communication or navigation techniques. These models are mainly used as mobility management schemes for mobile communication systems for predicting user positions[1]. There are various mobility models proposed for wireless sensor network(WSN). WSN is a group $\mathrm{N}$ sensor nodes in $A$. dynamic topologies consisting of 1 master node and $\mathrm{N}-1$ slave nodes; WSN works on data gathered mechanism where data from environment are collected and route to the processing node[20]. WSN application range consists of military application, industrial area, machine health monitoring, environmental, home automation, traffic control etc[21]. Mobility Models which are applicable for wireless sensor network are such as RDMM, GMMM, Manhattan, MOBAN etc. Implementation of mobility models using various routing protocols are done through simulation tools. Simulation tools, for studying of wireless sensor network which is develop in C, C++ or Java base language are QualNet, OPNET Modeler Wireless Suite, TOSSIM, OMNet++, NS-2, Avrora, J-Sim, ATEM, EmStar, SENS, MATLAB SIMULINK, ATEMU etc[16,21]. Each simulator tools state have its own merits and demerits[12].Performance evaluation of particular mobility model for WSN can be perform through simulating tools by applying routing protocols with different parameters. Routing protocol are the set of rules for the node movements in the networks[8] wireless sensor nodes which follow dynamic topologies also follows protocols to route the data gathered to the central processing node by using simulator[2,3,7].Performance metrics are parameters which are applied during experiments to evaluate the performance parameters such as throughput, velocity, packet delivery ratio, loss ratio, hop count, latency etc model with varying speed, node in network.
II. MOBILITY MODEL

Model which refer to movement of user along with the change in features such as location, velocity etc in network topologies over time, such model known as mobility models.

Mobility Models for wireless sensor networks are categorized under various head as shown : \section{A.}

Random Walk Mobility Model

In Random Walk Mobility Model, mobile nodes moves from its current location to a new target location randomly with new direction and speed. This new speed and direction are both chosen from predefined ranges, respectively [min-speed, max-speed] and $[0,2 *$ pi] respectively based on uniform distribution[2].

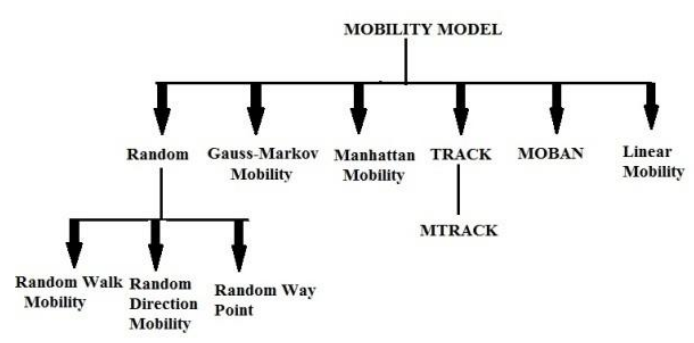

Fig. 1. Mobility Model

\section{B. Random Direction Mobility Model}

Random Direction Mobility Model modeled for concentration avoidance of mobile user at center of the area. In this model, mobile users pick a random direction to travel, users then travels to the border of the simulation area. Once the simulation boundary is reached, the mobile user halt for certain time then select direction in specific angle i.e., between 0 to 180 degree and then continue again in selected angle direction[2]. 


\section{Random Waypoint model}

The Random Waypoint Mobility Model make use of halt time due to the change in speed or direction or the combination of both. Movement of mobile user begins with start point by remaining halt for a certain period of time. Once this halt time expires, the mobile user moves to random end point in the simulation area. Speed here is equally distributed between [min-speed, max-speed] value [2].

\section{Gauss-Markov Mobility Model}

In the Gauss-Markov Mobility Model each mobile user is initialized with a speed and direction with fixed intervals of time movement occurs for updating the speed and direction of each node. Value of speed and direction at the nth instance of time is calculated based on the value of speed and direction at the $n-1$ st instance and a random variable [10]. Mandar Karyakarte et.al.[3] elaborates the equations for calculating speed and direction in detail.

\section{E. Manhattan Grid model}

The Manhattan mobility model uses a grid topology in which mobile users are allowed to move only in horizontal or vertical direction. At each intersection of a horizontal and a vertical street, the mobile users can turn left, right or go straight with certain probability[10].

\section{F. TRACK Mobility Model}

The TRACK Mobility Model is based on the Connected Dominating Set (CDS) property of a network graph. The CDS is developed using the localized Spanning Tree (MST) of the CDS is constructed using the length of edges as the weighing criteria[15].

\section{G. MTRACK Mobility Model}

The Multilevel-TRACK Mobility Model is the extended version of TRACK Mobility Model. The MTRACK Mobility Model is develop by exploiting the Connected Dominating Set (CDS) property of the network graph[15].

\section{H. Mobility Model for BODY AREA NETWORK}

MoBAN (Mobility Model for BANs) is a configurable mobility model for simulating wireless body area networks. The model is useful for simulating both intra- WBAN, where communication between various node in network and extra-WBAN protocols, where communications among a WBAN and its environment[24].

\section{Linear Mobility Model}

In linear mobility model, mobile user moves in a straight line in certain angle and this angle changes only when the mobile user hits a wall which reflects off the wall at the same angle.[7]

\section{ROUTING PROTOCOL}

Routing protocol specifies how routers communicate with one another, for widely scattering information that enables them to select routes between any two nodes in network topology. Following are the routing protocols reviewed among various referenced papers:

\section{A. Ad Hoc on Demand Distance Vector (AODV):}

AODV is distance vector type routing protocol that does not maintain routes that are not active up to end point . It uses Route request (RREQ), Route replies (RREPs), Route error(ReRR) messages to discover and maintain routes. When node wants a route to the end point it broadcasts RREQ to entire network till end point reached, then RREP is send back to the start point with discovered path. When node detect that route is invalid then it broadcast a RERR message.

\section{B. Destination Sequenced Distance Vector Routing Protocol(DSDV).}

DSDV is table driven algorithm based on Bellman-Ford routing. Each node has a routing table having the end point, next hop and number of hops to the end point. The nodes periodically broadcast updates in network. Shortest path is also identified simultaneously with sequence number tagging. Sequence number is updated to odd number when node detects route to end point is broken then hop number is set to infinity; while even number represents that path is well connected.

\section{SIMULATION TOOLS}

Tools used for implementing mobility models for WSN with different parameters for measuring performance in order to make reliable and perfect installment known as simulation tools. Following are simulating tools which reviewed during our survey :

\section{A. NS2 (Network Simulator 2)}

It cannot simulate more than 100 nodes and the problem of bandwidth and power consumption in WSN[2,9,10,12].

\section{B. OMNet++}

It supports MAC and some localized protocol and can simulate power consumptions and channel control[4,5,24,11,12].

\section{J-Sim}

It can simulate up to 500 sensor nodes, radio channels and power consumptions .It has longer execution time[22,23,12].

\section{MATLAB/Simulink}

It can simulate, analyze and model WSN in different topologies[21].

\section{E. OPNet}

It can run as discrete, event ,mobility model etc and there is no special routing protocol for WSN available as well as at least different modulation technique as well as ZigBee MAC layer is provided[20,21]. 


\section{PERFORMANCE METRICS}

Response of AODV and DSDV routing protocols on mobility models for WSN is evaluated through various performance metrics which are reviewed as below:

\section{A. Throughput(tp)}

Number of bits received by sink from various different sensor nodes. It is calculated using number of packets received at sink and size of each packet. Whereas kbps is a measuring unit for Throughput[2].

\section{B. $\quad$ Packet Delivery Function( $p d f)$}

Ratio of number of data packets received at sink denoted by to total number of data packets sent by various sensor node. The metric value range will varies between 0 to 1 , with higher value of showing better performance[2].

\section{C. $\quad$ Average End to End Delay(eed)}

Average of sum of transmission delay of each packet received at sink. The transmission delay of each packet can be measured as difference between time at which the $i^{\text {th }}$ packet was received at sink and time at which the packet was sent at source node. Smaller the value of average end to end delay shows better performance. Measuring unit of average end to end delay measured in seconds[2].

\section{Packet Loss Ratio(plr)}

Ratio of number of packets lost to the number of packets sent[7].

\section{LITERATURE REVIEW}

Applications of WSN for different mobility models such as RDMM, RWMM and Group Mobility Models built in NS2 simulator based on static and dynamic cluster algorithm. Author investigated that the dynamic based target tracking is acceptable for correct tracking with high energy consumption than static based clustering[1]. GMMM performance is better in random movement when compare with other mobility models such as RWMM, RDMM, RWPMM etc using AODV protocol [2].

Mobility Models such as RWMM, RWPMM, RDMM, Group Mobility Model, Pursue Mobility Model etc performances are evaluated through OMNet++ simulation tool using AODV protocol with packet delivery ratio, latency and throughput parameters are experimented with different speed for individual node[3]. Author investigate that position based routing protocols are better as compare to non-position based routing protocols with respect to packet delivery ratio and packet loss by performing comparative study for mobility models with various parameter using $\mathrm{OMNeT}++$ simulation tool with INET Framework [4].

Effects of mobility models for WSN with single sensor on traffic patterns are performed with model like random walk, random waypoint, and discrete Brownian motion etc [5]. These Mobility models are categorized in homogenous and heterogeneous model with underlying suitable model name was reviewed by researchers [6] .

Researchers presented comparative study of various mobility models such as RWPMM, GMMM, RPGMM, Manhattan Mobility Model ; using AODV and DSDV routing protocol with performance metrics such as throughput, velocity, hop count[7]. Routing protocols for WSN has various criteria[8].

Effect of mobility models over various routing protocols in WSN using NS2 simulation tool with parameter such as delay, routing load on variation of speed , node etc were analyzed[9]. As, Mobility models was classified as temporal dependency, spatial dependency, geographic factor etc [10]

Efficiency of WSN with respect of task management, energy consumption etc can be perform with the introduction of smart antenna in central node where experimented in OMNeT++ simulation tool[11]. However , there are various simulation tools for WSNs, which researchers can make selection as per requirement. A survey regarding different simulation tools along with their characteristics are presented[12].

A review about various mobility model Random models, Social models and Hybrid models and there further classification show that each model works as there characteristic in WSNs[13]. Author setup performance comparison among various mobility models with metrics such as End to End delay, Packet Loss probability, throughput etc by using OMNET++ simulation tools . They found generalized model with better output [14].

WSN implementation on TRACK and MTRACK mobility models that make use of Minimum Spanning Tree of connected dominating set for sensor node by enhancing the performance of the network by using optimization technique[15]. Hardware support for building Wireless Sensor Network and configuration setup about sensor node, radio modules as well as about various simulation tools which is compatible with WSN are clarified[16].

Research regarding paper on Health monitoring application of Wireless Sensor network based on mobility aware and energy efficient protocols[17]. IPv6 protocol over Low Power Wireless Personal Area Networks (6LoWPAN) WSN for improving location based mobility performance was studied[18].

Extended Ad hoc On-Demand Distance Vector (EAODV) routing protocol that dynamically changes the node mobility as tree structure for WSN the performance is analyses using NS2 simulation tool with loss rate, throughput, energy consumption and delay metrics are analyzed [19].Continuous based mobility model generates 
better performance with metrics such as energy comparison of both AODV and DSDV routing protocols consumption packet delivery etc then continuous and with different performance metrics state that GMMM has event based mobility model with different ZIGBEE maximum value then Random Model variations and routing and topologies by using OPNET simulation Manhattan. However, AODV routing protocol have tool[20]. MATLAB/Simulink Simulation tool for higher throughput and less loss ratio then DSDV routing implementing WSN .Researcher state that this simulation protocol[2,7].

method is good for studying the effect of channel noise and interference, Signal to noise ratio etc. which are physical layer parameter while MATLAB/Simulink tool is also flexible in building the end nodes and sensors[21].

Simulating tool name J-Sim and NS-2 of wireless sensor network which is geographically routed by Greedy Perimeter Stateless Routing algorithm that uses the technique of forwarding the packet based on closest node and perimeter mode performance are comparatively studied different factor such as number of events, simulation with completion time, memory etc[22-23]. In case of memory allocation scenario J-Sim found efficient then NS-2.

Among the various application of WSN; MOBAN has been an interesting and vast research area for WBAN. OMNeT++ simulation tool with mixim framework by integrating WBAN communication protocols and applications by which performance evaluation of MOBAN undertaken[24] .One of most Challenging part for wireless sensor networks are how to maintain network life with sensors, this challenging task solution has been investigated by using mobility-assisted data collection (MADC) model with various network parameter[25]

\section{VII.}

\section{PERFORMANCE OF VARIOUS MOBILITY MODEL}

Performance of user movement in wireless sensor network can be measure through parameter metrics which are analyzed under routing protocols as follows:

TABLE I

MOBILITY MODEL PERFORMANCE

\begin{tabular}{|c|c|c|c|c|c|}
\hline \multirow{2}{*}{ S.No } & \multirow{2}{*}{$\begin{array}{c}\text { Mobility } \\
\text { Model }\end{array}$} & \multicolumn{4}{|c|}{ Parameter } \\
\cline { 3 - 6 } & & tp & pdf & plr & eed \\
\cline { 3 - 6 } & & Min & Min & ----- & ----- \\
\hline 1. & RWMM & AODV \& \\
DSDV
\end{tabular}

Mandar Karyakarte et.al. and R.Alagu Pushpa et.al. tested above model in NS2 and OMNet++ simulation tools which are identified in survey that,

\section{CONCLUSION}

Mobility models play vital role for the node movement in WSN. It is important for picking suitable routing protocol with various performance parameters in WSN simulation tools for achieving desired results. Thus, during our survey we have reviewed various experimental papers by which in future, point regarding improvement in efficiency of WSN with different metrics are clarified. Finally, survey regarding interaction of mobility models with WSN was accomplished.

\section{ACKNOWLEDGMENT}

This work is supported by School of Studies in Computer Science \& I.T , Pt. Ravi Shankar Shukla University, Raipur, India.

\section{REFERENCES}

[1] Aysegul Alaybeyoglu, Orhan Dagdeviren, Kayhan Erciyes, Aylin Kantarci " Performance Evaluation of Cluster-based Target Tracking Protocols for Wireless Sensor Networks " 978-1-42445023-7/09 @2009 IEEE.

[2] Mandar Karyakarte, Anil Tavildar \& Rajesh Khanna " Effect Of Mobility Models On Performance Of Mobile Wireless Sensor Networks " International Journal of Computer Networking, Wireless and Mobile Communications (IJCNWMC)ISSN 22501568 Vol. 3, Issue 1, Mar 2013, 137-148 @ TJPRC Pvt. Ltd.

[3] S H Manjula, C N Abhilash, Shaila K, K R Venugopal, L M Patnaik " Performance of AODV Routing Protocol using Group and Entity Mobility Models in Wireless Sensor Networks" In Proceedings of the International MultiConference of Engineers and Computer Scientists 2008 Vol II IMECS 2008, 19-21 March, 2008, Hong Kong.

[4] Atta ur Rehman Khan, Shahzad Ali, Saad Mustafa, Mazliza Othman " Impact of mobility models on clustering based routing protocols in mobile WSNs ". On 2012 10th International Conference on Frontiers of Information Technology.

[5] Pu Wang and Ian F. Akyildiz " Effects of Different Mobility Models on Traffic Patterns in Wireless Sensor Networks " In IEEE Globecom 2010 proceedings.

[6] Anas Abu Taleb, Tareq Alhmiedat, Osama Al-haj Hassan, Nidal M. Turab " A Survey of Sink Mobility Models for Wireless Sensor Networks " Journal of Emerging Trends in Computing and Information Sciences" $\quad$ Vol. 4, No. 9 September 2013 ISSN 2079-8407.

[7] R.Alagu Pushpa, A.Vallimayil, V.R.Sarma Dhulipala " Impact of Mobility models on Mobile Sensor Networks ". International Journal of Communication Network \& Security, Volume-1, Issue-1, 2011.

[8] Shio Kumar Singh, M P Singh and D K Singh " A Survey-Routing Protocols in Wireless Sensor Networks " International Journal of Computer Science \& Engineering Survey (IJCSES) Vol.1, No.2, November 2010.

[9] G. Santhosh Kumar, M.V. Vinu Paul and K. Poulose Jacob " Impact of Node Mobility on Routing Protocols for Wireless Sensor Networks " In International Conference on Sensors and Related Networks (SENNET'07), VIT University, Vellore, India. Dec. 1214, 2007. pp.480-485.

[10] Vasnthi.V, Romen Kumar.M, Ajith Singh.N, And M.Hemalatha "A Detailed Study of Mobility Models in Wireless Sensor Network" Journal of Theoretical and Applied Information Technology November 2011. Vol. 33 No.1.

[11] Kerem Kucuk , Adnan Kavak , Halil Yigit " A Smart Antenna Module Using OMNeT++ for Wireless Sensor Network Simulation " 1-4244-0979-9/07 IEEE ISWCS 2007. 
[12] Fei Yu, Prof. Raj Jain "A Survey Of Wireless Sensor Network Simulation Tool" http://www1.cse.wustl.edu/ jain/cse56711/ftp/sensor/index.html.sdwd 23XZ.

[13] Michal Gorawski, Krzysztof Grochla " Review of Mobility Models for Performance Evaluation of Wireless Networks ".

[14] Sh. Al-Shukri , B. Sriram , R. B. Lenin and S. Ramaswamy "A System of Systems Approach: A Benchmark to WSNs Mobility Models " On 2010 5th International Conference on System of Systems Engineering.

[15] Avinash Srinivasan, Jie Wu " TRACK: A Novel Connected Dominating Set based Sink Mobility Model for WSNs".

[16] Andhe Dharani, Shantharam Nayak , Manjuprasad B "Hardware and Simulation Perspective Study on Wireless Sensor Networks". International Journal of Advance Foundation and Research in Computer (IJAFRC) Volume 1, Issue 7, July 2014. ISSN 2348 - 4853.

[17] Youssouf Zatout, Rahim Kacimi , Jean-François Llibre and Eric Campo " Mobility-aware Protocol for Wireless Sensor Networks in Health-care Monitoring" In 5th IEEE Workshop on Personalized Networks (PerNets 2011).

[18] Xiaonan Wang, Deguang Le, Yufeng Yao, Conghua Xie " Location-based mobility support for 6LoWPAN wireless sensor networks " Journal of Network and Computer Applications 49 (2015) 68-77.

[19] De-gan Zhang, Xiao-dong Song, Xiang Wang, Yuan-ye Ma " Extended AODV routing method based on distributed minimum transmission (DMT) for WSN" In Int.J.Electron.Commun.(AEÜ)69(2015)371-381.

[20] Dusan Stevanovic , Natalija Vlajic "Performance of IEEE 802.15.4 in Wireless Sensor Networks with a Mobile Sink Implementing Various Mobility Strategies 0022 " 978-1-4244-2413-9/08 @2008 IEEE.

[21] Qutaiba I. Ali " Simulation Framework of Wireless Sensor Network (WSN) Using MATLAB/SIMULINK Software "http://dx.doi.org/10.5772/46467.

[22] Ahmed Sobeih, Wei-Peng Chen, Jennifer C. Hou , Lu-Chuan Kung, Ning Li, Hyuk Lim, Hung-Ying Tyan and Honghai Zhang " J-Sim: A Simulation Environment for Wireless Sensor Networks " In Proceedings of the 38th Annual Simulation Symposium (ANSS'05) 1080-241X/05 \$20.00 (C) 2005 IEEE.

[23] Ahmed Sobeih, Wei-Peng Chen , Jennifer C. Hou , Lu-Chuan Kung, Ning Li , Hyuk Lim, Hung-Ying Tyan and Honghai Zhang " J-SIM: A SIMULATION AND EMULATION ENVIRONMENT FOR WIRELESS SENSOR NETWORKS " In IEEE Wireless Communications • August 2006.

[24] Majid Nabi, Marc Geilen, Twan Basten " MoBAN: A Configurable Mobility Model for Wireless Body Area Networks ".

[25] Wang Liu, Kejie Lu, Senior Member, IEEE, Jianping Wang, Guoliang Xing, Member, IEEE, and Liusheng Huang "Performance Analysis of Wireless Sensor Networks With Mobile Sinks" In IEEE transactions on vehicular technology, Vol.61, No.6, July 2012. 